\title{
A Dihydroflavonol-4-Reductase-Like Protein Interacts with NFR5 and Regulates Rhizobial Infection in Lotus japonicus
}

\author{
Liujian Duan, ${ }^{1}$ Junqing Pei, ${ }^{1}$ Yaping Ren, ${ }^{1}$ Hao Li, ${ }^{1}$ Xiangzhen Zhou, ${ }^{1}$ Hui Zhu, ${ }^{1,+}$ Deqiang Duanmu, ${ }^{1}$ \\ Jiangqi Wen, ${ }^{2}$ Kirankumar S. Mysore, ${ }^{2}$ Yangrong Cao, ${ }^{1}$ and Zhongming Zhang ${ }^{1, \dagger}$ \\ ${ }^{1}$ State Key Laboratory of Agricultural Microbiology, College of Life Science and Technology, Huazhong Agricultural University, \\ Wuhan, 430070, China; and 2Plant Biology Division, The Samuel Roberts Noble Foundation, Ardmore, OK 73401, U.S.A.
}

Accepted 4 October 2018.

\begin{abstract}
In almost all symbiotic interactions between rhizobia and leguminous plants, host flavonoid-induced synthesis of Nod factors in rhizobia is required to initiate symbiotic response in plants. In this study, we found that Lotus japonicus Nod factor receptor 5 (LjNFR5) might directly regulate flavonoid biosynthesis during symbiotic interaction with rhizobia. A yeast two-hybrid analysis revealed that a dihydroflavonol-4-

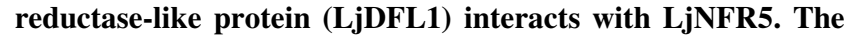
interaction between MtDFL1 and MtNFP, two Medicago truncatula proteins with homology to LjDFL1 and LjNFR5, respectively, was also shown, suggesting that interaction between these two proteins might be conserved in different legumes. $L j D F L 1$ was highly expressed in root hairs and epidermal cells of root tips. Lotus ljdflI mutants and Medicago mtdfl1 mutants produced significantly fewer infection threads (ITs) than the wild-type control plants following rhizobial treatment. Furthermore, the roots of stable transgenic $L$. japonicus plants overexpressing $L j D F L 1$ formed more ITs than control roots after exposure to rhizobia. These data indicated that $\mathrm{LjDFL1}$ is a positive regulator of symbiotic signaling. However, the expression of $L J D F L 1$ was suppressed by rhizobial treatment, suggesting that a negative feedback loop might be involved in regulation of the symbiotic response in L. japonicus.
\end{abstract}

Nitrogen-fixing symbiosis between rhizobia and legume plants starts with the exchange of signaling molecules between these two partners. Legume flavonoids released into the rhizosphere are recognized by compatible rhizobia through constitutively expressed NodD proteins, which induce the transcription of Nod factor (NF) synthesis-related genes (Kelly et al. 2018; Mulligan and Long 1985). Nod factors are then recognized by compatible legume NF receptors, such as

${ }^{\dagger}$ Corresponding authors: Z. Zhang; E-mail: zmzhang@mail.hzau.edu.cn and H. Zhu; E-mail: zhuhui@mail.hzau.edu.cn

Funding: This work was supported by the National Key R\&D Program of China (2016YF0100700), the National Natural Science Foundation of China (31670240 and 31870219), and the State Key Laboratory of Agricultural Microbiology (AMLKF201503, AMLKF201608).

*The $\boldsymbol{e}$-Xtra logo stands for "electronic extra" and indicates that eight supplementary figures and one supplementary table are published online.

(c) 2019 The American Phytopathological Society
LjNFR1 and LjNFR5 from Lotus japonicus (Madsen et al. 2003; Radutoiu et al. 2003) and MtLYK3 and MtNFP from Medicago truncatula (Arrighi et al. 2006; Limpens et al. 2003; Smit et al. 2007), to activate the symbiotic signaling followed by the formation of infection threads (ITs) and nodule organogenesis (Ibáñez et al. 2017; Madsen et al. 2010; Oldroyd et al. 2011). LjNFR1 and LjNFR5 form a heteromeric complex at the plasma membrane (Madsen et al. 2011), which can directly bind to Nod factors at high-affinity binding sites (Broghammer et al. 2012). Isothermal titration calorimetry and circular dichroism spectroscopy experiments suggest that the LysM2 domain of LjNFR5 is required for binding to Nod factors, which might cause a conformational change in the LysM2 domain (Sørensen et al. 2014).

Flavonoids are biologically active secondary metabolites synthesized by plants. More than 10,000 different flavonoids have been identified (Ferrer et al. 2008; Weston and Mathesius 2013). Flavonoids are important in many biological activities, such as flower coloration, protection against phytopathogens, and plant response to UV light (Falcone Ferreyra et al. 2012). Some flavonoids could regulate auxin transport by modulating the activity of ABCB-type auxin transporters and influencing the cycling, expression, and localization of PIN proteins (Bailly et al. 2008; Di Pietro et al. 2002; Geldner et al. 2001; Peer et al. 2004; Santelia et al. 2008). Flavonoids are critical regulators of auxin transport and accumulation during the formation of indeterminate nodules in Medicago truncatula ( $\mathrm{Ng}$ et al. 2015; Wasson et al. 2006; Zhang et al. 2009). A few specific flavonoids, known as isoflavonoids, act as pivotal infection signals leading to the synthesis of Nod factors in rhizobia. In addition to inducing the transcription of compatible rhizobial nod genes, flavonoids could alter the rhizobial surface polysaccharide content and cause numerous protein changes (Cooper 2007). Some distinct classes of flavonoids have been identified, such as methoxychalcone and medicarpin from Medicago sativa (Liu and Murray 2016). However, the isoflavonoids produced by L. japonicus, which induce Nod factor synthesis in Mesorhizobium loti, remain unknown.

The biosynthetic pathways resulting in the formation of numerous flavonoids, including certain isoflavonoids, have been resolved, and many flavonoid synthetases have been identified. Flavonoid biosynthesis begins with the phenylpropanoid pathway, which converts phenylalanine into 4-coumaroyl-CoA. Although 4-coumaroyl-CoA and malonyl-CoA are common raw materials in this biosynthesis pathway, various enzymes, including reductases, isomerases, hydroxylases, and monooxygenases, alter the flavonoid skeleton, resulting in different 
flavonoids with different physiological activities (Falcone Ferreyra et al. 2012; Martens et al. 2010). In the medicarpin biosynthesis pathway, a legume-specific enzyme, chalcone reductase (CHR) converts 4-coumaroyl-CoA and malonyl-CoA into isoliquiritigenin, which is then converted into flavanone by a novel isoform of chalcone isomerase (Liu and Murray 2016). Medicarpin is synthesized after a series of enzyme reactions by isoflavonoid-specific enzymes, including 2-hydroxyisoflavanone synthase, 2-hydroxyisoflavanone dehydratase, isoflavanone- $O$ methyltransferase, isoflavone-2'-hydroxylase, isoflavone reductase, vestitone reductase, and 7,2'-dihydroxy-4'-methoxy-isoflavonol dehydratase (Wang 2011).

LjNFR5 is an atypical protein kinase with the predicted activation loop missing in its kinase domain (Madsen et al. 2003). Although LjNFR5 has been identified for more than 15 years (Madsen et al. 2003; Radutoiu et al. 2003), its regulatory mechanisms controlling rhizobial infection and nodulation are largely unclear. Only a few proteins have been identified to be a downstream target of NFR5 to mediate symbiotic signaling. LjRop6 was identified as an interactor of LjNFR5 to regulate nodule organogenesis and Nod factor-induced root-hair deformation (Ke et al. 2012, 2016). In this study, we found that a flavonoid reductase, L. japonicus dihydroflavonol-4-reductaselike protein (LjDFL1), which is an interacting protein with LjNFR5, plays a positive role in regulating rhizobial infection. Since LjDFL1 is an enzyme involved in flavonoid biosynthesis, our data suggest a model in which perception of Nod factor might regulate flavonoid biosynthesis via the LjNFR5-LjDFL1 protein complex.

\section{RESULTS}

\section{Identification of $\mathrm{LjDFL1}$ as an interacting protein of LjNFR5.}

In L. japonicus, LjNFR1 and LjNFR5 are Nod factor receptors required for mediating symbiotic signaling in response to rhizobia (Madsen et al. 2003; Radutoiu et al. 2003). To identify the downstream target proteins of Nod factor receptors, we used the kinase domain of LjNFR5 (LjNFR5-KD) as a bait to screen a cDNA library reported previously (Zhu et al. 2008); 11 independent putative interacting colonies were obtained and sequenced. Ten of them encode the same protein that has a high level of amino acid sequence similarity to L. japonicus dihydroflavonol-4-reductase (LjDFR2) (GenBank accession number AAV71171), whose function is involved in flavonoid biosynthesis. We named this gene LjDFL1 (LjDFR2-like protein 1) (Supplementary Fig. S1).

The full-length cDNA of LjDFLl (GenBank accession number MH325020, Lotus Base v3 accession number Lj0g3v0327049) contains a 1,074-bp open reading frame encoding a protein of 357 amino acids. The deduced protein has a predicted isoelectric point of 7.05 and a molecular mass of $39.3 \mathrm{kDa}$. Protein domain analysis revealed that LjDFL1 contains an entire FR_SDR_e (flavonoid reductase, extended short-chain dehydrogenases/reductases) domain. Flavonoid reductases participate in the NADP-dependent reduction of flavonoids, which are ketone-containing plant secondary metabolites. LjDFL1 has active site characteristic of the SDRs and an NADP-binding motif.

Phylogenetic analysis revealed the presence of LjDFL1 homologs in monocotyledons and dicotyledons as a single-copy gene in diploid plants (Fig. 1). In legumes, LjDFL1 homologs form a distinct phylogenetic group (Fig. 1). Protein alignment analysis showed a high degree of sequence conservation in the NADP binding site and a substrate binding. LjDFL1 and MtDFL1 both have the Rossmann fold, which has an alphabeta-alpha folding pattern. Predicted three-dimensional models showed that both LjDFL1 and MtDFL1 have ten alpha-helices and nine beta-sheets.

\section{LjNFR5 associates with LjDFL1.}

We performed a yeast two-hybrid assay to confirm the interaction between LjDFL1 and LjNFR5. Yeast cells containing LjDFL1 and LjNFR5-KD were able to grow on synthetic defined (SD)/-Leu/-Trp/-Ade/-His medium, but no growth was observed when the yeast cells were cotransformed with LjNFR1-KD and LjDFL1 or with LjNFR5-KD and LjDFR1. These data indicate that LjDFL1 but not LjDFR1 specially interacts with the kinase domain of LjNFR5 but not of LjNFR1 (Fig. 2A; Supplementary Fig. S2).

To test the effect of protein structure integrity on the interaction between LjNFR5 and LjDFL1, we expressed several truncated proteins of LjDFL1 fused to the Gal4 activation domain and several truncated proteins of LjNFR5-KD fused to the Gal4 DNA binding domain in yeast cells. The truncated LjDFL1 proteins either did not interact with LjNFR5-KD or they had a very weak interaction. Except for LjNFR5 (325 to 595), the truncated proteins of LjNFR5-KD did not interact with LjDFL1, suggesting that full-length LjDFL1 and LjNFR5 (325 to 595) are required for their interaction in yeast cells.

To provide further evidence that LjNFR5 interacts with LjDFL1, we performed in vitro protein pull-down, coimmunoprecipitation (Co-IP), and bimolecular fluorescence complementation (BiFC) assays. We expressed His-tagged LjNFR5-KD in Escherichia coli and purified the recombinant protein using Ni-affinity beads. We then incubated maltosebinding protein (MBP)-tagged LjDFL1 immobilized on amylose resin with purified LjNFR5-KD. After repeated washing with buffer, the proteins retained on the beads were eluted and were separated by sodium dodecyl sulfate-polyacrylamide gel electrophoresis (SDS-PAGE). As shown in Figure 2C, LjNFR5$\mathrm{KD}$ was retained on the MBP-LjDFL1 beads, while it was not possible to pull down LjNFR5-KD with beads containing MBPLjDFR1. These data confirmed that LjDFL1 physically interacts with LjNFR5-KD.

For the Co-IP assay, hemagglutinin (HA)-tagged LjDFL1 and FLAG-tagged LjNFR5 and LjNFR1 were coexpressed in Nicotiana benthamiana leaves, driven by the LjUbql promoter. Proteins precipitated on an anti-FLAG M2 affinity gel were extracted and probed with anti-HA antibody and anti-FLAG antibody, respectively. As shown in Figure 2D, LjDFL1 coimmunoprecipitated with LjNFR5 but LjNFR1 was not coimmunoprecipitated in the anti-FLAG M2 affinity gel, indicating that LjDFL1 interacts with LjNFR5 but not LjNFR1.

For the BiFC assay, LjDFL1 fused with the C-terminal half of yellow fluorescence protein (YFPC) and LjNFR5 or LjNFR1 fused with the N-terminal half of yellow fluorescence protein (YFPN) were coexpressed in $N$. benthamiana leaf cells. Expression of the recombinant proteins was verified by immunoblotting with horseradish peroxidase-conjugated anti-HA or anti-Myc antibodies (Supplementary Fig. S3). When YFPC-LjDFL1 and LjNFR5YFPN were coexpressed in $N$. benthamiana leaves, strong yellow fluorescence signals were observed on the plasma membrane (Fig. 3A to C). However, no fluorescence was observed when LjNFR1-YFPN and LjDFL1-YFPC were coexpressed in $N$. benthamiana leaves (Fig. 3D to F), confirming that LjDFL1 interacts with LjNFR5 but not LjNFR1.

\section{The interaction between NFR5 and DFL1 homologs is conserved in legumes.}

To test whether the interaction between DFL1 and NFR5 homologs is conserved among legumes, orthologs of LjDFL1 and LjNFR5 were cloned from Medicago truncatula. MtNFP is the ortholog of LjNFR5 (Arrighi et al. 2006). In a yeast 
two-hybrid assay, we showed that the kinase domain of MtNFP (MtNFP-KD) indeed interacts with MtDFL1 and LjDFL1 and MtDFL1 also interacts with LjNFR5-KD (Fig. 2B). BiFC assays gave similar results; MtDFL1 interacts with both MtNFP and LjNFR5 and LjNFR5 also interacts with MtDFL1 (Fig. 3G to $\mathrm{O})$. These results suggest that interaction between NFR5 and DFL1 homologs is conserved among legumes.

\section{LjDFL1 is expressed at high levels in root tips.}

To characterize the temporal and spatial expression of $L j D F L 1$, we used quantitative reverse transcription-polymerase chain reaction (qRT-PCR) to measure the transcript levels of LjDFL1 in roots, nodules, leaves, stems, flowers, and pods (Fig. 4A). $L j D F L 1$ transcripts were detected in all tested tissues, with the highest level of expression in root tissues. We also examined the expression of $L j D F R$ genes, using qRT-PCR (Fig. 4A). $L j D F R 1$ transcripts were detected at higher levels in roots and nodules than in the other tissues analyzed. $L j D F R 2$ and $L j D F R 5$ were found mostly in flowers and pods, while LjDFR4 mRNA was detected in leaves, stems, flowers, and pods.

To determine the pattern of $L j D F L 1$ expression at the cellular level in roots, a promoter fragment of $L j D F L 1$ of approximately

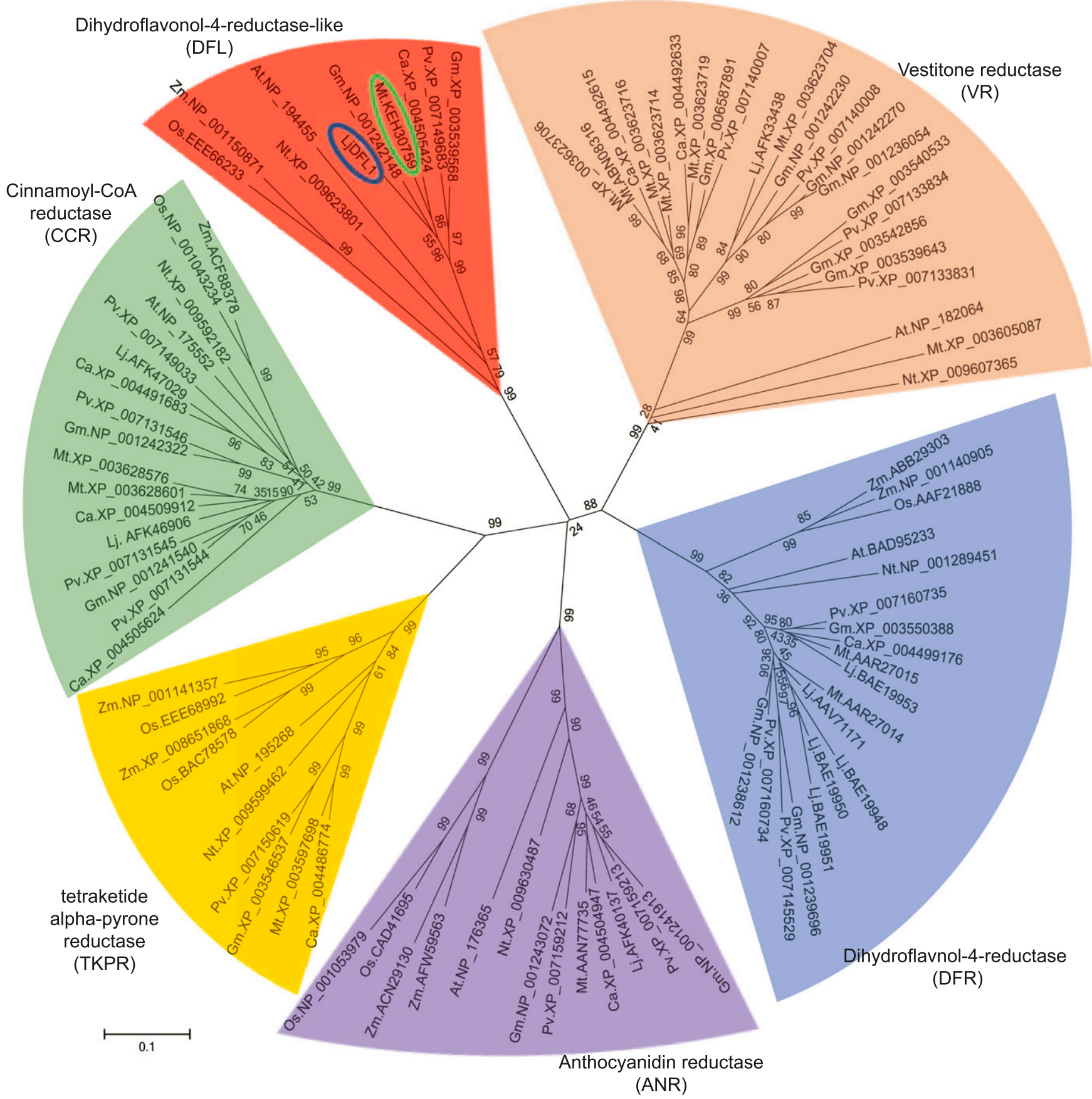

Fig. 1. Phylogenetic tree of dihydroflavonol-4-reductase-like proteins (DFLs), dihydroflavonol-4-reductases (DFRs), anthocyanidin reductases (ANRs), tetraketide alpha-pyrone reductases (TKPRs), cinnamoyl-CoA reductases (CCRs), and vestitone reductases (VRs). LjDFL1 is marked with a blue oval MtDFL1 (Mt.KEH30759) is marked with a green oval. Six clades representing DFLs (red), DFRs (blue), ANRs (purple), TKPRs (gold), CCRs (green), and VRs (orange) are indicated. The phylogenetic tree shows the phylogenetic relationships among DFLs from Lotus japonicus, Medicago truncatula, Glycine max, Phaseolus vulgaris, Cicer arietinum, Arabidopsis thaliana, Nicotiana tomentosiformis, Oryza sativa japonica group, and Zea mays. Bootstrap values (\%) obtained from 1,000 trials are indicated for each node. Neighbor-joining phylogenetic analysis was accomplished using MEGA software. 
$2 \mathrm{~kb}$ in length was cloned and was used to drive the expression of the $\beta$-glucuronidase (GUS) reporter in transgenic hairy roots. In uninoculated and inoculated roots, GUS staining revealed that $L j D F L 1$ was highly expressed in root tips, especially in the root hairs near the root tip, as well as in the epidermal cells of the elongation and maturation zones of root tips (Fig. 5C to F). GUS activity was also observed in the root cap, epidermal cells of the meristematic zone of root tips, and the vascular tissue of roots (Fig. 5A and B).

To further confirm the expression of $L j D F L 1$ in the root tip, we performed a qRT-PCR assay to assess the expression levels of $L j D F L 1$ in 1.5-cm-long root tips and in roots without tips. The expression of $L j D F L 1$ in 1.5-cm-long root tips was two to three times as high as that in roots without tips (Fig. 5G), while the positive control gene NIN was significantly induced (Fig. 5H).

\section{Rhizobial treatment downregulates $L j D F L 1$ expression.}

LjDFL1 was expressed at much lower levels in roots inoculated with $M$. loti at 14 days postinoculation (dpi) than in uninoculated roots (Fig. 4A), suggesting that $L j D F L 1$ expression was downregulated in roots inoculated with $M$. loti. To confirm this, samples of uninoculated roots or roots inoculated with $M$. loti taken at different timepoints postinoculation were collected for qRT-PCR analysis. LjDFL1 expression was significantly downregulated in roots inoculated with rhizobia compared with uninoculated roots (Fig. 4B); however, LjDFRl expression was not significantly downregulated (Fig. 4C). Expression of LjDFL1 in roots 0.5 to 5 dpi with rhizobia declined by 48 to $80 \%$ compared with that in the corresponding uninoculated roots.

To test whether LjDFL1 expression declined in roots treated with Nod factors, crude purified Nod factors from $M$. loti (López-Lara et al. 1995) were applied to L. japonicus roots at different timepoints postinoculation. LjDFL1 expression declined gradually over time (Fig. 4D). In the L. japonicus mutants $n f r 5$, symrk, ccamk, nsp2, and nin, which failed to establish symbiosis, LjDFL1 expression was not significantly decreased after rhizobial inoculation (Fig. 4E), suggesting that downregulation of $L j D F L 1$ expression might be related to the symbiotic signaling pathway.

We next examined whether mutation of the M. loti nod gene would influence $L j D F L 1$ expression in inoculated roots. We obtained
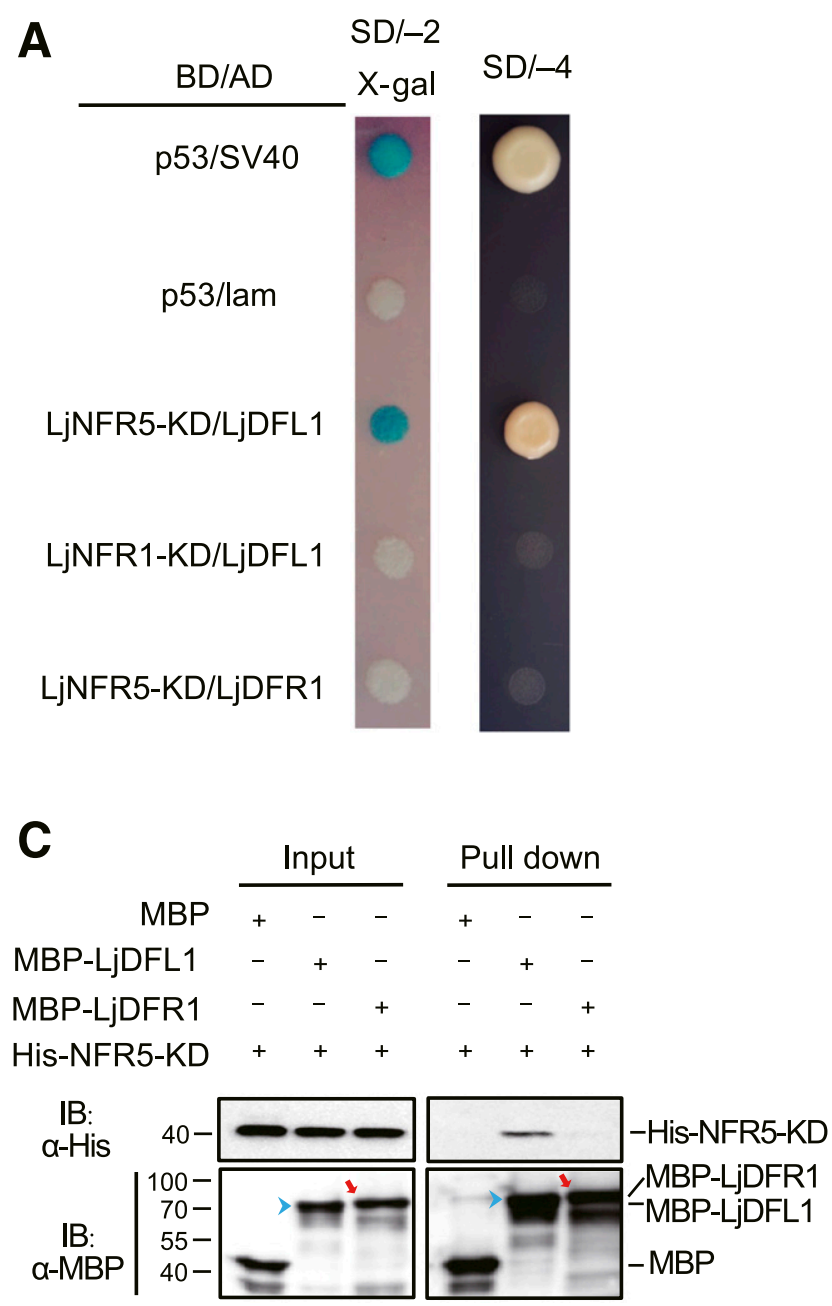

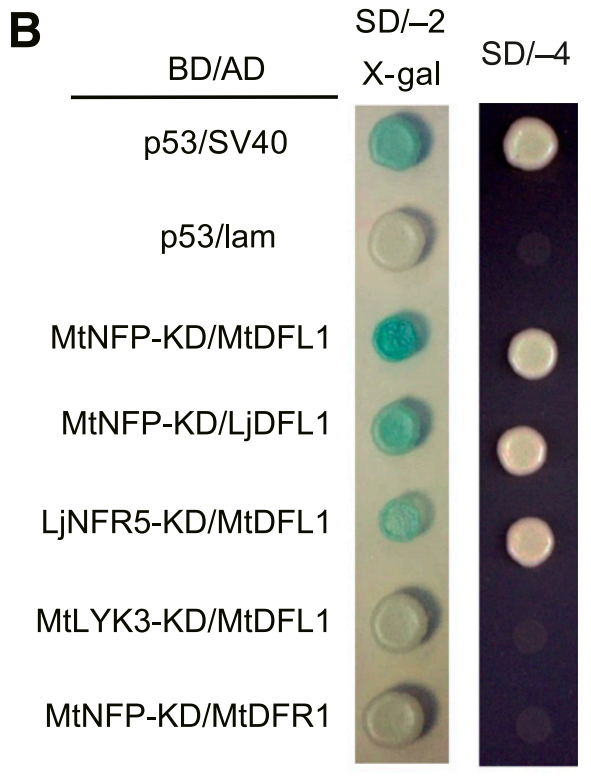

D

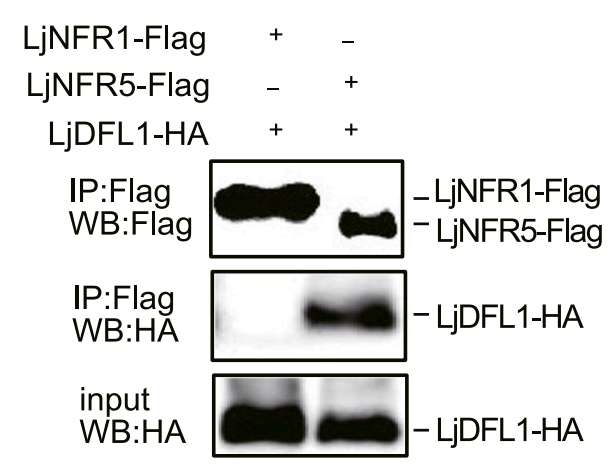

Fig. 2. Yeast two-hybrid assays, pull-down assays, and coimmunoprecipitation assays between LjNFR5 and LjDFL1. A, Yeast two-hybrid assays between the kinase domain of LjNFR5 (LjNFR5-KD) and LjDFL1. The positive control was p53/SV40, and the negative control was lam/SV40. Synthetic defined (SD)/-2 $\mathrm{X}$-gal = SD/-Leu/-Trp containing $80 \mu \mathrm{g} \mathrm{ml}{ }^{-1} \mathrm{X}-\mathrm{gal}$; SD/-4 = SD/-Leu/-Trp/-Ade/-His. B, Yeast two-hybrid assays between LjNFR5-KD (or MtNFP-KD) and dihydroflavonol-4-reductase-like proteins (DFLs). C, In vitro protein-protein interaction assays between LjDFL1 and LjNFR5-KD. Purified His-NFR5-KD was mixed with maltose-binding protein (MBP) alone or immobilized MBP-LjDFL1 or MBP-LjDFR1 fusion protein. After washing, proteins pulled down by amylose resins were separated by sodium dodecyl sulfate-polyacrylamide gel electrophoresis (SDS-PAGE). The gels were used for immunoblot analysis with anti-His-tag antibody (top) or anti-MBP-tag antibody (bottom). Blue arrow arrowheads indicate MBP-LjDFL1, while red arrows indicate MBP-LjDFR1. D, Coimmunoprecipitation of LjDFL1 and LjNFR5. Hemagglutinin (HA)-tagged LjDFL1 and Flag-tagged LjNFR5 or LjNFR1 proteins were coexpressed in Nicotiana benthamiana leaf cells. Input samples were detected using anti-HA antibody (bottom). Immunoprecipitates were separated on SDS-PAGE gels and were probed with anti-HA antibody (middle) or anti-FLAG antibody (top). 
an $M$. loti MAFF303099 nodC knockout mutant by triparental mating method. Since nodC encodes $N$-acetylglucosaminyltransferase, which is required for Nod factor synthesis, we used this mutant as a Nod factor-deficient strain to treat plants. We assessed the expression of LjDFL1 and NPL (Xie et al. 2012) in roots inoculated with wild-type (WT) M. loti or nodC mutant 3 dpi with rhizobia, using qRT-PCR. As a control assay, the rhizobia nodC mutant strain did not induce the expression of NPL (Fig. 4G), while LjDFL1 was expressed at much higher levels in roots inoculated with the M. loti MAFF303099 nodC mutant than in roots inoculated with WT M. loti (Fig. $4 \mathrm{~F})$. This suggests that Nod factors might suppress LjDFL1 expression.

\section{LjDFL1 plays a positive role during rhizobial infection.}

To obtain further insight into the role of LjDFL1 in the symbiotic interaction between M. loti and L.japonicus, we used CRISPR/Cas9 technology to obtain L. japonicus LjDFL1 knockout mutants (Wang et al. 2016). Two gene-specific guide sequences were designed to target the first and second exon of $L j D F L 1$, respectively (Supplementary Fig. S4). T2 generation plants of the LjDFL1 mutant were isolated from stable transgenic plants with the CRISPR/Cas9-induced LjDFL1 mutation. A 1-bp insertion in exon 1 led to early termination of translation in the ljdfl1-1 mutant, whereas a 1-bp deletion near the PAM1 site and a 2-bp deletion near the PAM2 site that led to the change of 23 amino acids of the LjDFL1 protein was identified in the ljdfll-2 mutant. The nodulation phenotype was compared between wild type and ljdfl mutant plants 5 dpi with $M$. loti MAFF303099 constitutively expressing LacZ (Kang et al. 2011). The numbers of ITs per plant and per centimeter of root were significantly lower in ljdfll-1 and ljdfll-2 than in the WT MG20 plants (Fig. 6A and B). However, no significant differences in the numbers of nodule primordia (NPs) per plant and per centimeter of root were observed between control and mutant plants.

To test whether the expression of early nodulation genes, including $N I N, N P L$, and $N F-Y A 1$, was affected in ljdfll mutants, qRT-PCR was performed to measure the transcript levels of these genes in ljdfll and the WT roots at $5 \mathrm{dpi}$. The expression of NIN, NPL, and NF-YAI was decreased in the ljdfl 1 mutant roots compared with the WT roots (Supplementary Fig. S7). These data indicated that loss of LjDFL1 function leads to fewer ITs during infection in L. japonicus.

Considering the positive role of LjDFL1 in rhizobial symbiosis, we sought to identify the function of MtDFL1 during symbiosis. We used a PCR-based reverse screening method to identify MtDFL1 mutants from the Noble Foundation Medicago truncatula mutant collection (Cheng et al. 2014; Tadege et al. 2008). We obtained two independent mutant lines with a Tnt1 insertion in MtDFL1. The mutant lines NF11624 and NF19403 carry a Tnt 1 insertion at 23 and 22 bp after the ATG in the first exon, respectively (Supplementary Fig. S5). WT plants and homozygous mutants were identified from the T2 mutants of NF11624 and NF19403 with PCR, using genomic DNA as template. MtDFL1 transcript levels in both homozygous mtdfl1 mutant lines were not detected using qRT-PCR with primers MtDFL1-RT1F and MtDFL1-RT1R but were decreased by 82 to $91 \%$ compared with WT roots with primers MtDFL1-RT2F and MtDFL1-RT2R, suggesting a small amount of the mRNA of LjDFL1 fused to Tnt1 exists in both homozygous mtdfl1 mutant roots.

Homozygous mutant plants and WT offspring from heterozygous mutant or Medicago R108 WT plants were grown in nitrogen-free soil and were observed at 4 dpi with Sinorhizobium meliloti 2011 expressing lacZ. The numbers of ITs and NPs per plant in both homozygous mutant lines were significantly lower than in the WT offspring of the heterozygous mutant or R108 plants. The number of ITs per plant was 9.4 in R108 plants, 9.4 in WT sibling plants harboring the mtdfll-1 allele, 3.9 in mtdfl1-1 (NF11624 homozygous mutant lines) plants, 11.9 in WT sibling plants harboring the $m t d f l 1-2$ allele, and 5.3 in mtdfl1-2 (NF19403 homozygous mutant lines) plants. The number of NPs per plant was 2.2 in R108, 2.6 in WT sibling plants with the $m t d f l 1-1$ allele, 0.9 in $m t d f l 1-1$ mutant plants, 2.8 in WT sibling plants with the $m t d f l 1-2$ allele, and 1.1 in $m t d f l 1-2$ plants.

We classified ITs into four groups based on the location of the IT tips (Supplementary Fig. S6), microcolony (MC), elongating IT in the root hair (eIT), IT reaching to the root epidermis (epIT), and ramified infection thread (rIT). The

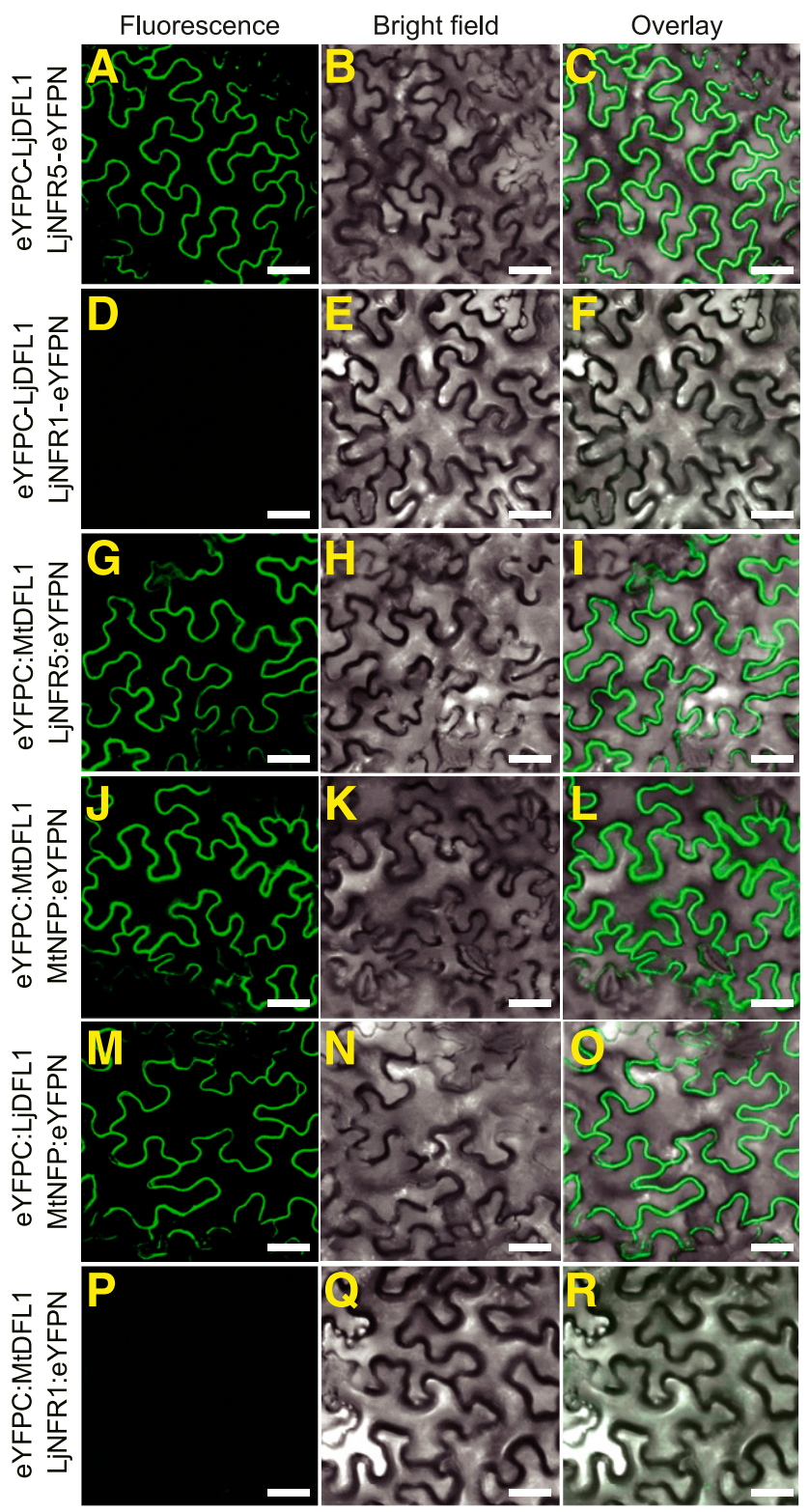

Fig. 3. Detection of protein-protein interactions in Nicotiana benthamiana leaves by bimolecular fluorescence complementation. A to R, Enhanced yellow fluorescent protein (eYFP) was split into eYFPC155 and eYFPN173 and was fused to LjDFL1 and LjNFR5, respectively. $N$. benthamiana leaves were cotransformed with eYFPC-LjDFL1 and LjNFR5-eYFPN and were observed via confocal microscopy. Strong fluorescence signals were observed between LjNFR5 and LjDFL1 (A to C), LjNFR5 and MtDFL1 (G to I), MtNFP and MtDFL1 ( $\mathrm{J}$ to L), and MtNFP and LjDFL1 (M to O), using a similar fusion approach. No fluorescence signals were detected between LjNFR1 and LjDFL1 (D to F) or LjNFR1 and MtDFL1(P to R). Bars = $50 \mu \mathrm{m}$. 

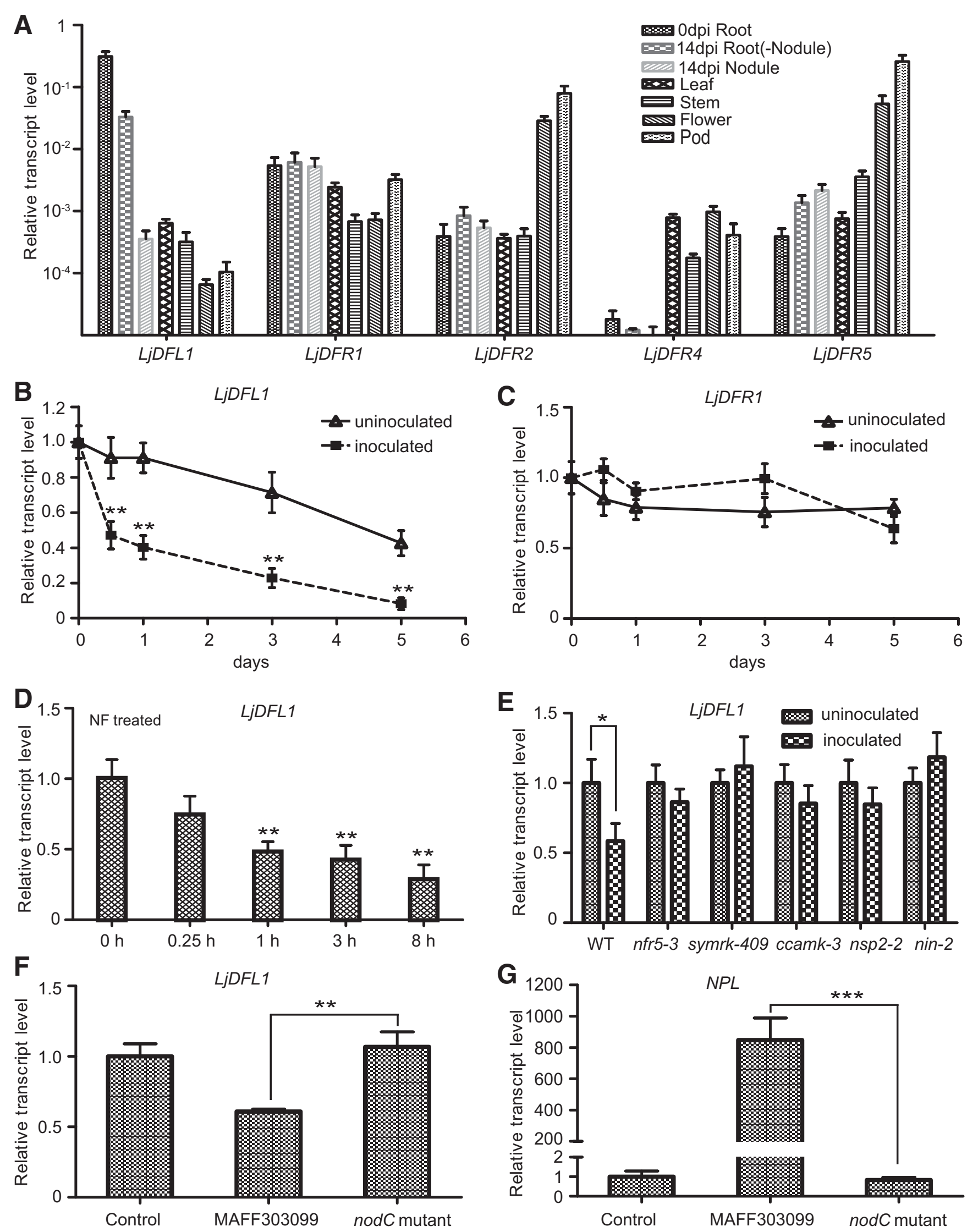
numbers of MCs, eITs, epITs, and rITs were dramatically reduced in mtdfll-1 compared with WT sibling plants with the $m t d f l 1-1$ allele. Similarly, the numbers of MCs, epITs, and rITs were significantly reduced in mtdfll-2 compared with WT sibling plants with the $m t d f l 1-2$ allele. These results indicate that mutation of MtDFL1 has deleterious effects on the formation and growth of ITs and NPs in Medicago truncatula.

To further study the biological function of $L j D F 1$ in the early infection process, we counted the number of ITs in the stable transgenic plants overexpressing $L j D F L 1$. Two stable transgenic $L$. japonicus line overexpressing $L j D F L 1$ under the LjUbql promoter were generated using Agrobacterium tumefaciens-mediated transformation. Plants transformed with the pUBHyg empty vector served as a control (Maekawa et al. 2008). Transcripts of LjDFLl were about 29-fold higher in LjDFL1-OX-2 and about 38-fold higher in LjDFL1-OX-8 than in control roots (Supplementary Fig. S8). LjDFL1-OX-2, LjDFL1OX-8, and control plants were inoculated with $M$. loti MAFF303099 constitutively expressing a lacZ marker and were observed at $5 \mathrm{dpi}$. The number of ITs per plant was 15.0 in the control, 25.1 in $L j D F L 1-\mathrm{OX}-2$, and 20.1 in LjDFL1-OX-8 (Fig. 6C), whereas the number of ITs per centimeter of root was 1.9 in the control, 3.1 in LjDFL1-OX-2, and 2.5 in LjDFL1-OX8 (Fig. 6D). These results demonstrate that the numbers of ITs per plant and per centimeter of root are significantly increased in $L j D F L 1-O X-2$ and LjDFL1-OX-8 compared with the control, whereas there were no significant differences in the numbers of NPs per plant or per centimeter of root (Fig. $6 \mathrm{C}$ and D). Gene expression analysis revealed that NIN, NPL, and $N F-Y A l$ expression increased significantly in $L j D F L 1$ overexpression roots compared with control roots. These results suggest that LjDFL1 promotes the formation of ITs in L. japonicus.

\section{DISCUSSION}

In almost all legume-rhizobial interactions, the compatible symbiosis begins with a dialog between legumes and rhizobia. Secretion of specific flavonoids by the host induces the biosynthesis of Nod factors by rhizobia. Then the perception of Nod factors by lysin motif (LysM) receptor kinases, such as LjNFR1/MtLYK3 and LjNFR5/MtNFP, initiates symbiotic signaling in the plant (Broghammer et al. 2012; Radutoiu et al. 2007). In this study, we cloned a gene encoding LjDFL1, an enzyme that might be involved in flavonoid metabolism. We demonstrated that LjDFL1 interacts with a Nod factor receptor, LjNFR5, to positively regulate rhizobial symbiosis in Lotus spp. However, $L j D F L 1$ expression was reduced after rhizobial or Nod factor treatment. Thus, we propose a model in which the Nod factor receptor associates with LjDFL1 to regulate symbiotic signaling. via the regulation of flavonoid metabolism, while downregulation of $L j D F L 1$ expression following rhizobial treatment seems to be a negative feedback of the symbiotic signaling pathway.

Flavonoids, the metabolites from the general phenylpropanoid pathway, mediate genotype-specific nodulation in legume-rhizobium symbiosis, since each rhizobium detects the specific flavonoids secreted by its compatible plant hosts to induce the synthesis of Nod factors. LjDFL1 encodes a protein containing an FR_SDR_e domain, which participates in the NADP-dependent reduction of ketone-containing plant secondary metabolites. MsVR (vestitone reductase), a protein with an FR_SDR_e domain, was reported to be involved in medicarpin biosynthesis in Medicago truncatula (Guo and Paiva 1995). MsVR catalyzes the conversion of vestitone to 7,2'dihydroxy-4'-methoxy-isoflavonol. LjDFL1 and MsVR belong to extended SDR subfamilies, which have a GXXGXXG cofactor-binding motif and a YXXXK active site motif (Kavanagh et al. 2008). LjDFL1 is such a protein with a high level of amino acid sequence similarity with L. japonicus LjDFR2, whose function is to catalyze the conversion of dihydroflavonols into leucodelphinidins during flavonoid biosynthesis. Since LjDFL1 positively regulates rhizobial infection, it is possible that it directly or indirectly modulates the biosynthesis of the specific flavonoids required for Nod factor synthesis in rhizobia, thereby allowing compatible symbiosis. Future research will aim to identify the specific substrate of LjDFL1 and the specific function of LjDFL1 in flavonoid metabolism.

Perception of Nod factors by two LysM kinases, such as L. japonicus NFR1 and NFR5, is required to mediate symbiotic signaling in response to rhizobia. An alreadyproposed model, LjNFR1 phosphorylates LjNFR5 and forms a receptor complex with LjNFR5 to initiate symbiotic signaling in L. japonicus. However, few downstream components of the receptor complex have been identified to date. LjDFL1 represents a novel downstream component of the Nod factor receptor complex. Furthermore, LjDFL1 seems to interact specifically with LjNFR5 but not LjNFR1, and this interaction is conserved in the symbiotic signaling pathways of different legumes. Since LjDFL1 is a putative enzyme involved in flavonoid biosynthesis and plays a positive role in symbiotic signaling, it is possible that perception of Nod factors by host plants might activate flavonoid biosynthesis to induce Nod factor biosynthesis by rhizobia and, thereby, promote further bacterial infection. This regulatory pathway also seems to be conserved in legumes, since, in both Medicago truncatula and L. japonicus, DFL1 interacts with NFR5 (or NFP) to positively regulate bacterial infection. However, whether the function of DFL1 in the symbiosis require its interaction with NFR5 remains to be elucidated.

The compatible interaction between rhizobia and legumes induces the development of nodules. The number of nodules is tightly controlled by host plants to avoid an excessive burden of nodules. The association of LjDFL1 with LjNFR5 suggests that Nod factor-mediated signaling might regulate flavonoid biosynthesis to induce the secretion of more Nod factors. However, downregulation of $L j D F L 1$ expression serves as negative feedback regulation to suppress symbiotic signaling, allowing a controlled degree of rhizobial infection. Our data suggest a novel mechanism that regulates symbiotic signaling in legumerhizobial symbiosis.

Fig. 4. Expression profiles of $L j D F L 1$ assessed by quantitative reverse transcription polymerase chain reaction. A, Expression patterns of $L j D F L 1, L j D F R 1$, LjDFR2, LjDFR4, and LjDFR5. RNA samples were acquired from Lotus japonicus uninoculated roots, the nodule-stripped roots at 14 days postinoculation (dpi) with Mesorhizobium loti, the nodules at $14 \mathrm{dpi}$, 3-week-old stems, leaves, flowers, and pods. B and C, Quantification of $L j D F L 1$ and $L j D F R 1$ expression in inoculated or uninoculated roots at the indicated timepoints. D, LjDFL1 expression in Nod factor-treated roots. E, $L j D F L 1$ expression in symbiosis-deficient mutant roots. Total RNA was extracted from symbiosis-deficient mutant and wild-type Gifu roots with $M$. loti inoculation at 3 dpi and from mock-inoculated roots. $\mathbf{F}$ and $\mathbf{G}, L j D F L 1$ expression in roots inoculated with $M$. loti nodC mutant. Total RNA samples were extracted from roots inoculated with $M$. loti or the $M$. loti nodC mutant 3 dpi with rhizobia. Roots of the same age that had not been inoculated served as control. $N P L$ expression was used as a marker gene (G). Error bars indicate \pm standard deviation from three biological replicates. Student's $t$ test, one asterisk $(*)$ indicates $P<0.05$, two (**) $P<0.01$, and three (***) $P<0.001$. 


\section{MATERIALS AND METHODS}

\section{Biological material and plant growth.}

WT L. japonicus Miyakojima MG-20, WT ecotype Gifu-129, and ecotype Gifu-129 loss-of-function mutants (nfr5-3, symrk409, ccamk-3, nsp2-2, nin-2) were used for qRT-PCR. symrk-409 contains an LORE1 insertion leading to the early termination of translation of SYMRK (Li et al. 2018). The seeds were surfacescarified in $\mathrm{H}_{2} \mathrm{SO}_{4}$ for 5 to $8 \mathrm{~min}$, followed by 8 to $12 \mathrm{~min}$ in $2 \%$ sodium hypochlorite. Medicago truncatula ecotype R108 and mutants were used for phenotype analysis. Seeds were surfacescarified in $\mathrm{H}_{2} \mathrm{SO}_{4}$ for 2 min, followed by sterilization with $2.5 \%$

\section{Uninoculated}
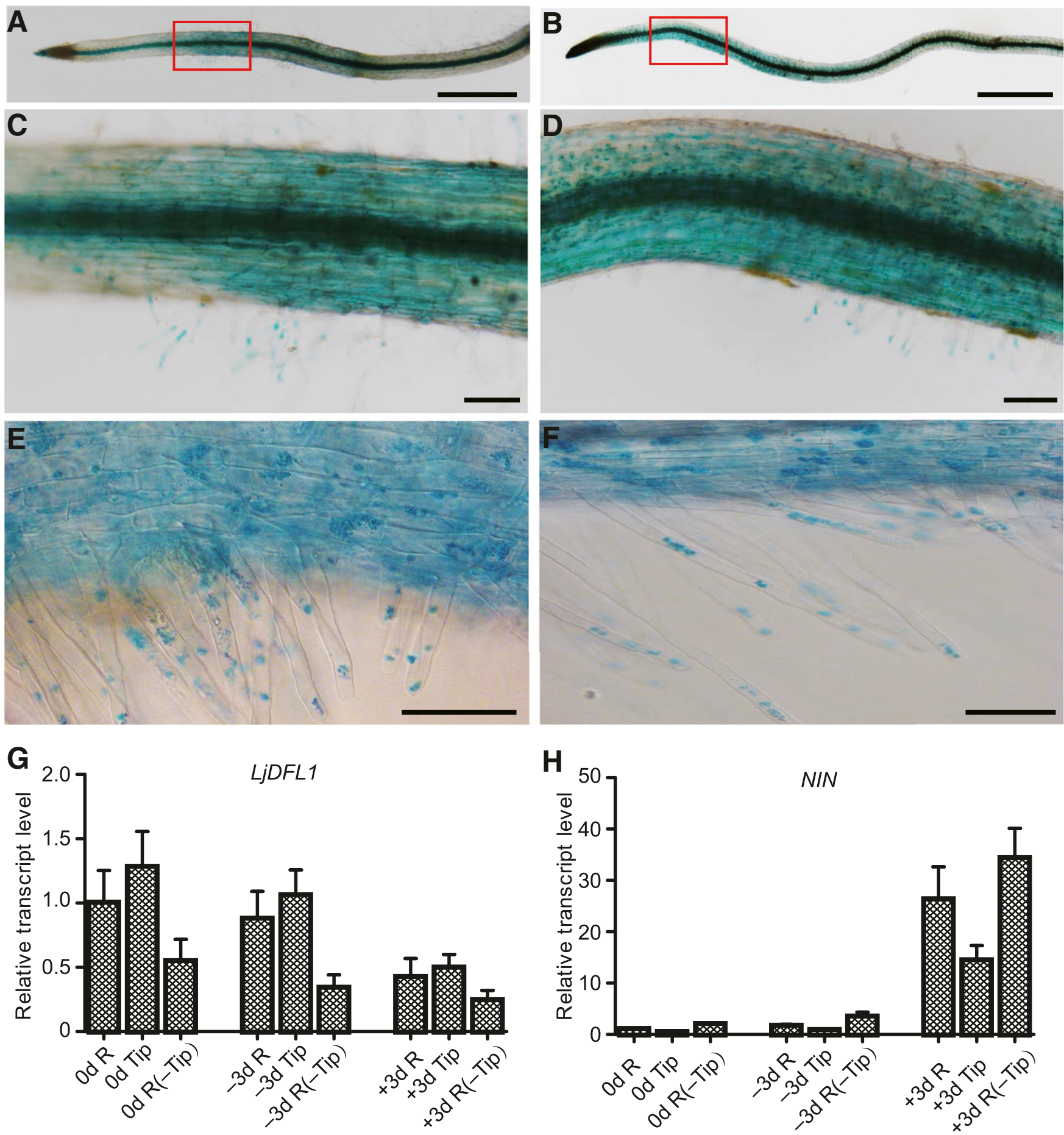

Fig. 5. Expression analysis of $L j D F L 1$ in roots. A to $\mathbf{F}$, Histochemical $\beta$-glucuronidase (GUS) staining of transgenic hairy roots expressing $L j D F L 1_{\text {pro }}$ : $G U S$. A to $\mathrm{D}$ are fluorescence stereomicroscopy images, whereas $\mathrm{E}$ and $\mathrm{F}$ are photographs obtained using a light microscope. $\mathrm{C}$ and $\mathrm{D}$ are enlargements of the regions enclosed in red boxes in A and B, respectively. Transgenic hairy roots were not inoculated (A, C, and E) or inoculated with Mesorhizobium loti at 3 days postinoculation (dpi) (B, D, and F). A and B, bars $=1 \mathrm{~mm} ; \mathrm{C}$ and D, bars $=100 \mu \mathrm{m} ; \mathrm{E}$ and F, bars $=50 \mu \mathrm{m}$. G and $\mathbf{H}$, Expression of $L j D F L 1$ and $N I N$ in root tips assessed by quantitative reverse transcription polymerase chain reaction. Total RNA was isolated from Lotus japonicus total roots (R), 1.5-cm-long root tips (Tip), or roots lacking the $1.5-\mathrm{cm}-$ long root tips $[\mathrm{R}(-\mathrm{Tip})]$, which were inoculated with $M$. loti and were analyzed at $3 \mathrm{dpi}(+3 \mathrm{~d})$ or not inoculated $(-3 \mathrm{~d})$. Uninoculated roots $(0 \mathrm{~d} \mathrm{R})$ served as a control. Expression of $N I N$ was used as a marker gene $(\mathrm{H})$. Error bars indicate \pm standard deviation from three biological replicates. 
of sodium hypochlorite for 5 to $8 \mathrm{~min}$. Germinated seeds were planted in pots filled with perlite and vermiculite $(1: 1 \mathrm{vol} / \mathrm{vol})$ supplied with a half-strength B\&D nitrogen-free medium (for L. japonicus seedlings) or Fahraeus medium (for Medicago truncatula seedlings) in growth cabinets with a 16-h light and 8-h dark cycle at $22 \pm 1^{\circ} \mathrm{C}$. Five-day-old seedlings were inoculated with M. loti MAFF303099 expressing lacZ (for L. japonicus seedlings) or $S$. meliloti 2011 constitutively expressing LacZ (for Medicago truncatula seedlings).

\section{Plant transformation and $N$. benthamiana leaf infiltration.}

Generation of transgenic hairy roots of L. japonicus with Agrobacterium rhizogenes LBA1334 was performed as described previously (Wang et al. 2016). Transgenic hairy roots expressing $L j D F L 1_{p r o}$ :GUS were used for histochemical GUS staining to analyze the pattern of $L j D F L 1$ expression in roots. GUS staining of the transgenic hairy roots was performed as described previously (Wang et al. 2015).
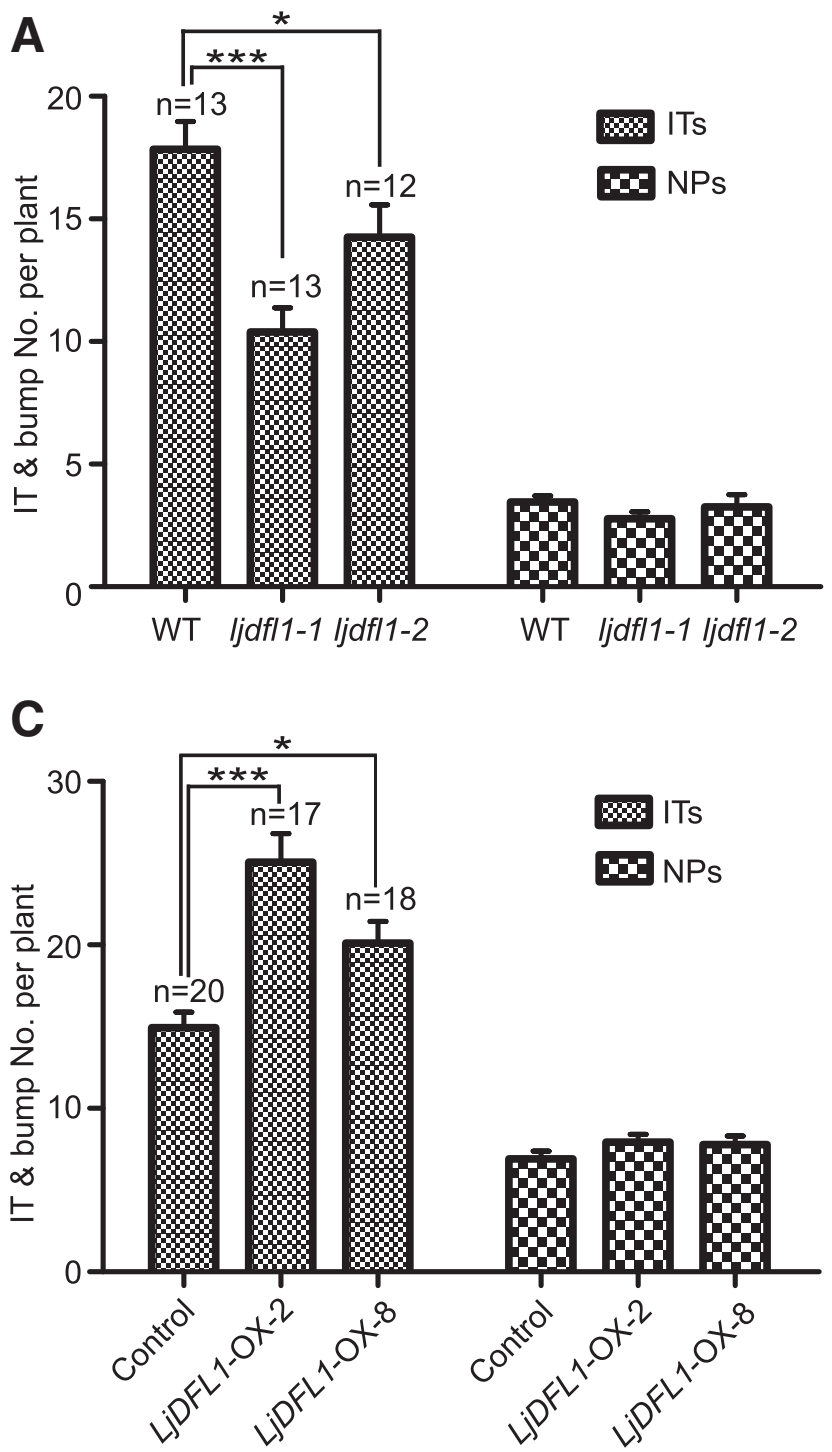

Stable transformation of $L$. japonicus with A. tumefaciens EHA105 was carried out as described previously (Wang et al. 2016). Hypocotyls of MG20 seedlings were infected with EHA105 harboring proper constructs. Calli were screened with hygromycin and the regenerated plants (T0) were grown to obtain the T1 seeds. A piece of cotyledon from every T1 seedling was used to extract genomic DNA. Positive plants were identified by PCR with the primers of the hygromycin gene. For stable transgenic plants of CRISPR/Cas9, PCR products with $L j D F L 1$ specific primers were sequenced to confirm CRISPR/Cas9 induced LjDFL1 mutation. For stable transgenic plants of $L j D F L 1$ overexpression, $L j D F L 1$ transcript levels were analyzed using qRT-PCR.

Infiltration of $N$. benthamiana leaves were performed as described previously (Kang et al. 2015). For BiFC, equal amounts of two strains of EHA105 harboring proper constructs were mixed and were used for infiltration and leaves were observed and photographed by a fluorescence stereo microscope
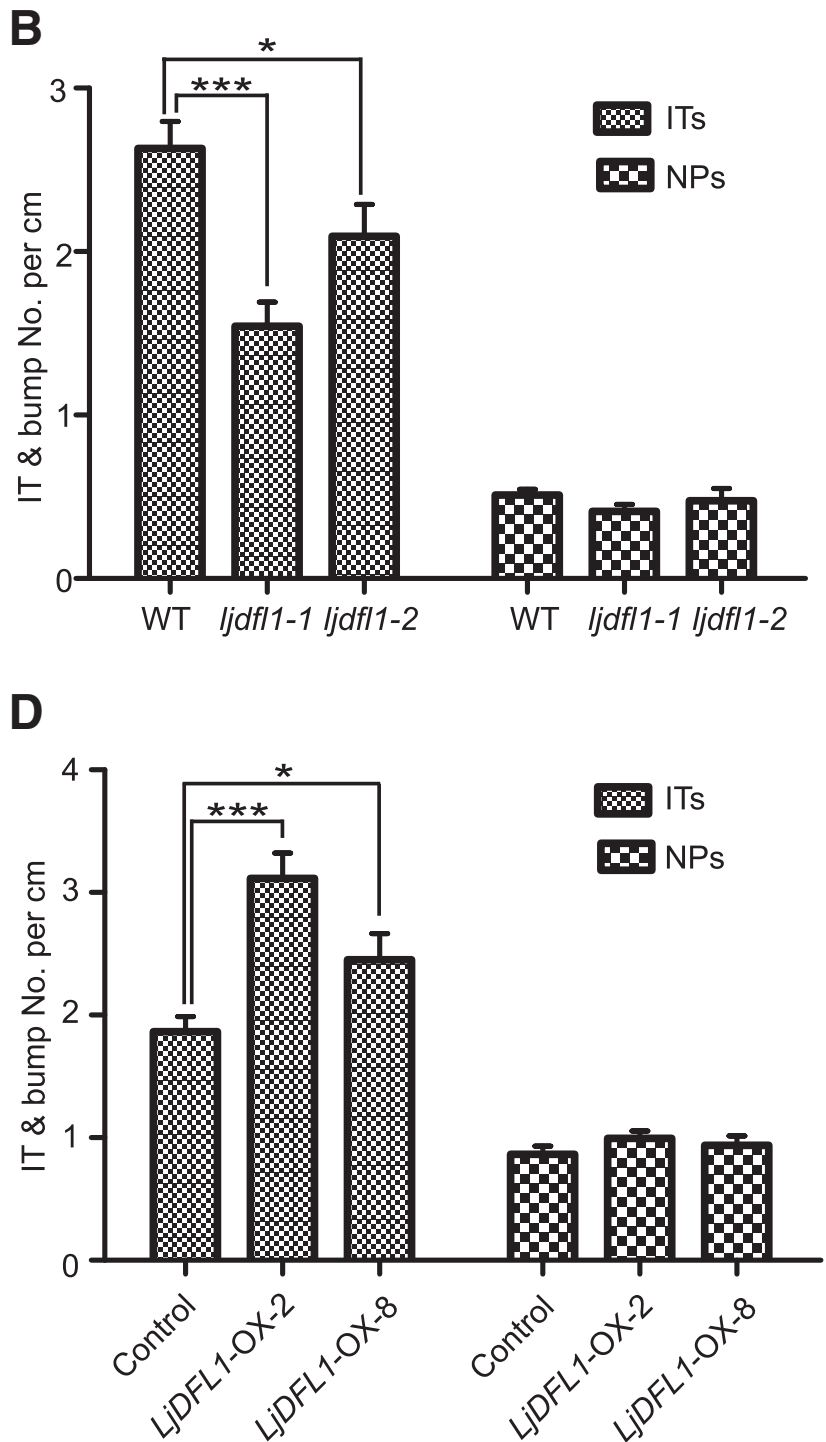

Fig. 6. Effect of LjDFL1 mutation and overexpression on rhizobial infection. Plants were grown in nitrogen-free soil and were observed 5 days postinoculation (dpi) with Mesorhizobium loti MAFF303099 constitutively expressing a lacZ marker. A and B, Stable transgenic plants with CRISPR/Cas9-induced $L j D F L 1$ mutation were generated by Agrobacterium tumefaciens-mediated transformation. T2 generation plants of the LjDFL1 mutant, ljdfl1-1 and ljdfl1-2, were assayed for their symbiotic phenotype. The numbers of infection threads (ITs) and nodule primordia (NPs) per plant (A) and per centimeter of root (B) were

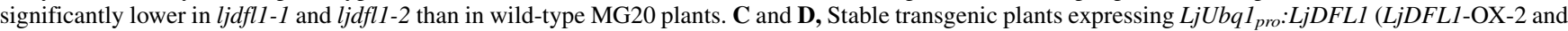
LjDFL1-OX-8) were obtained by A. tumefaciens-mediated transformation. Transgenic Lotus japonicus plants expressing the empty vector served as a control. The numbers of ITs and NPs per plant (C) and per centimeter of root (D) were significantly higher in $L j D F L 1$-OX-2 and $L j D F L 1$-OX-8 than in control plants. Student's $t$ test, one asterisk (*) indicates $P<0.05$ and three $(* * *) P<0.001$. 
(Olympus SZX16) 36 to $48 \mathrm{~h}$ after infiltration. YFP fluorescence was excited at $514 \mathrm{~nm}$ and detected at 522 to $558 \mathrm{~nm}$.

\section{Yeast two-hybrid screen.}

The cDNA fragment encoding the LjNFR5 (GenBank number AJ575255) kinase domain (272 to 595 amino acids) was amplified by PCR and was cloned into pGBKT7 vector for expressing the bait protein Gal4 DNA-binding domain BD: LjNFR5-KD. The bait plasmid (pGBKT7-LjNFR5-KD) was transformed into yeast strain Y187 by the lithium acetate method. Screening of the L. japonicus root cDNA library (Zhu et al. 2008) was carried out according to the manufacturer's instructions (Clontech). For testing protein-protein interactions in yeast cells, a prey construct plasmid and a bait construct plasmid were transformed into yeast strains AH109 and Y187, respectively. After mating of the two strains, colonies grew on the SD/-Leu/-Trp medium and, then, were transferred to SD/-Leu/-Trp/X-gal and SD/-Leu/-Trp/-Ade/-His medium for testing protein-protein interactions.

\section{Plasmid construction.}

For the promoter-GUS fusion, the 2-kb promoter of $L j D F L 1$ with the $5^{\prime}$ untranslated region was amplified by PCR and was cloned into the BamHI/SmaI site of DX2181GFPa vector. For yeast two-hybrid assays, cDNAs encoding LjNFR5-KD, LjNFR1-KD, MtNFP-KD, MtLYK3-KD, LjDFL1, LjDFR1, and the LjNFR5-KD truncated forms were amplified by PCR and were cloned into the EcoRI/SmaI site of pGBKT7 to produce fusion proteins with the GAL4-DNA binding domain. To produce fusion proteins with the GAL4 activation domain, cDNAs encoding LjDFL1, LjDFR1, MtDFL1, MtDFR1, LjNFR5-KD, LjNFR1-KD, and the LjDFL1 truncated forms were amplified and cloned into the EcoRI/SmaI site of pGADT7, respectively. For in-vitro interaction assays, the LjDFL1 and LjDFR1 cDNAs were amplified and inserted into the EcoRI/SalI site of pMAL-c2X for expression of MBPLjDFL1 and MBP-LjDFR1, respectively. The LjNFR5-KD cDNA fragment was amplified and cloned into the EcoRI/SalI site of pET30a for expression of His-tagged LjNFR5-KD recombinant protein. For Co-IP analysis, the LjNFR1 and LjNFR5 cDNAs without the stop codon were amplified and inserted into the $X b a \mathrm{I} / K p n \mathrm{I}$ site of pUBGFP N-3×Flag for expressing LjNFR1-flag and LjNFR5-Flag, respectively. The LjDFL1 cDNA without the stop codon was amplified and cloned into the $X b a \mathrm{I} / K p n \mathrm{I}$ site of pUBGFP $\mathrm{N}-3 \times \mathrm{HA}$ for expressing LjDFL1-HA. These constructs were introduced to A. tumefaciens EHA105 for infiltration. For BiFC analysis, the LjDFLland MtDFL1 cDNAs were amplified and cloned into the SalI/SmaI site of pSPYCE(MR) (Waadt et al. 2008) vector for expressing YCM-(HA)-LjDFL1 and YCM-(HA)-MtDFL1, respectively. The $L j N F R 1, L j N F R 5$, and MtNFP cDNAs without the stop codon were amplified and inserted into the SalI/SmaI site of pSPYNE173 to obtain LjNFR1-(myc)-YN, LjNFR5(myc)-YN, MtNFP-(myc)-YN fusions. The constructs were transferred into $A$. tumefaciens EHA105 for infiltration. For gene knockout analyses, DNA fragments of $2 \times 35$ S-Cas 9 and two gene-specific guide RNA sequences driven by the $L j U 6$ promoter were cloned into pCAMBIA1300 vector as described previously (Wang et al. 2016). For overexpression of LjDFL1, the LjDFL1 cDNA was cloned into the $X b a \mathrm{I} / A s c \mathrm{I}$ site of pUBHYG (Maekawa et al. 2008), which contained a hygromycin selection marker for A. tumefaciens-mediated stable transformation. For $M$. loti nodC mutant construction, the gentamicin resistance gene was amplified and inserted into the EcoRI/SmaI site of pK18mobsacB (Schäfer et al. 1994) to obtain plasmid pK18mobsacB-Genta. Two partial DNA fragments of nodC were cloned into the EcoRI/AscI site and the SpeI/SmaI site of
pK18mobsacB-Genta in turn to obtain plasmid pK18mobsacBnodC-up-Genta-down.

\section{In-vitro protein-protein interactions.}

To assay the interaction between LjDFL1 and LjNFR5-KD in vitro, MBP-tagged LjDFL1 or LjDFR1 were absorbed on the amylose resins and were then mixed with $20 \mu \mathrm{g}$ of purified HisNFR5-KD protein in $1 \mathrm{ml}$ of interaction buffer $(20 \mathrm{mM}$ Tris- $\mathrm{HCl}$, $100 \mathrm{mM} \mathrm{NaCl}, 50 \mathrm{mM} \mathrm{KCl}, 1 \mathrm{mM} \mathrm{MgCl}$, $20 \mathrm{mM}$ Tris- $\mathrm{HCl}$, $0.5 \mathrm{mM}$ EDTA, and 5\% glycerol, $\mathrm{pH} 7.4$ ) on ice for $1 \mathrm{~h}$ with gentle shaking. The suspension $(100 \mu \mathrm{l})$ was used as the input sample and was mixed with SDS loading buffer. The rest of the amylose resins were washed 10 times, each, with $1 \mathrm{ml}$ of interaction buffer. The retained proteins were eluted by boiling in $1 \mathrm{w} \times$ SDS loading buffer and were separated on SDSPAGE gels. The interactions were assayed by immunoblotting with anti-MBP-tag antibody and anti-His-tag antibody (Promoter Biotechnology Ltd.) as described previously (Wang et al. 2015).

\section{Co-IP analysis.}

HA-tagged LjDFL1 and FLAG-tagged LjNFR5 or LjNFR1 were coexpressed in $N$. benthamiana leaves by coinfiltration of two Agrobacterium EHA105 strains containing proper constructs. Two days after infiltration, leaves were collected and were powdered in liquid nitrogen and crude proteins were extracted on ice for $20 \mathrm{~min}$ by a $1 \mathrm{~g}$ to $1 \mathrm{ml}$ volume ratio of leaf to homogenization buffer (50 mM HEPES, pH 7.5, $150 \mathrm{mM}$ $\mathrm{NaCl}, 5 \mathrm{mM}$ EDTA, $10 \%$ sucrose, $1.5 \%$ Triton $\mathrm{X}-100,1 \times$ cocktail protease inhibitor [Roche] and $2 \mathrm{mM}$ phenylmethylsulfonyl fluoride [Sigma], added before use). Samples were centrifuged at $13,000 \times g, 4^{\circ} \mathrm{C}$ three times for $20 \mathrm{~min}$ each, and the supernatants were incubated with ANTI-FLAG M2 affinity gel (Sigma) on ice for $2 \mathrm{~h}$ with gentle shaking. A 30- $\mu$ l sample was used as the input sample and was mixed with SDS loading buffer. The retained samples were washed five times with washing buffer $(50 \mathrm{mM}$ HEPES, pH 7.5, $10 \mathrm{mM}$ EDTA, $150 \mathrm{mM} \mathrm{NaCl}$, and $0.5 \%$ Triton X-100) to remove nonspecific binding. The samples were resuspended in $100 \mu \mathrm{l}$ of $1 \times$ SDS loading buffer, were boiled for $10 \mathrm{~min}$, and were separated on SDS-PAGE gels. Proteins were transferred to the polyvinylidene diflouride membrane (Bio-Rad) for Western blot analysis with antiFLAG and anti-HA antibodies (Promoter Biotechnology Ltd.).

\section{Construction and screening of $M$. loti nodC mutants.}

E. coli $\mathrm{S} 17-1$ and $\mathrm{HB} 101$ strains were used as hosts for the mobilizable plasmid pK18mobsacB-nodC-up-Genta-down and the helper plasmid pRK2073 (Leong et al. 1982), respectively. Double crossover events were generated in the nodC of WT M. loti MAFF303099. M. loti MAFF303099 was grown in tryptone yeast extract medium at $30^{\circ} \mathrm{C}$. E. coli was grown at $37^{\circ} \mathrm{C}$ in Luria-Bertani medium, supplemented with gentamicin (40 mg/liter) and spectinomycin (100 mg/liter) for S17-1 donor cells and HB101 helper cells, respectively. pK18mobsacBnodC-up-Genta-down plasmid was transformed into $M$. loti using triparental mating (Shimoda et al. 2008; Simon et al. 1983). Cells were plated on SM selection medium (per liter: $10 \mathrm{~g}$ of mannitol, $0.5 \mathrm{~g}$ of $\mathrm{KH}_{2} \mathrm{PO}_{4}, 0.2 \mathrm{~g}$ of $\mathrm{MgSO}_{4} \cdot 7 \mathrm{H}_{2} \mathrm{O}$, $0.1 \mathrm{~g}$ of $\mathrm{CaCl}_{2} \cdot 6 \mathrm{H}_{2} \mathrm{O}, 20 \mathrm{mg}$ of $\mathrm{H}_{3} \mathrm{BO}_{3}, 20 \mathrm{mg}$ of $\mathrm{Na}_{2} \mathrm{MoO}_{4}$, $10 \mu \mathrm{g}$ of thiamine, $10 \mu \mathrm{g}$ of calcium pantothenate, $10 \mu \mathrm{g}$ of nicotinamide, $1 \mu \mathrm{g}$ of Biotin) supplemented with gentamicin (40 mg/liter), phosphonomycin (100 mg/liter), and 10\% sucrose. Colony PCR was used to screen the nodC mutants with two pairs of primers, nodC-902bp-UP-F and Genta-94bp-R 
and Genta-481bp-F and nodC-769bp-down-R (Supplementary Table S1).

\section{qRT-PCR analysis.}

Total RNAs from roots and leaves were isolated using Trizol reagent (Invitrogen), and total RNAs from nodules, stems, flowers, and pods were isolated using Trizol Plants total RNA isolation kit (TransGen Biotech). The RNA concentration of total RNAs was measured at $260 \mathrm{~nm}$ with a NanoDrop 2000 (Thermo). The Primescript RT reagent kit (Takara) was used to eliminate genomic contamination and synthesize first-strand cDNAs. Quantitative PCR was performed on an ABI ViiATM 7 real-time PCR system using the SYBR Select master mix reagent (ABI). All PCR reactions were carried out under the standard cycling mode: $50^{\circ} \mathrm{C}$ for $2 \mathrm{~min}, 10 \mathrm{~min}$ at $95^{\circ} \mathrm{C}$, followed by 40 cycles of $15 \mathrm{~s}$ at $95^{\circ} \mathrm{C}$ and $1 \mathrm{~min}$ at $60^{\circ} \mathrm{C}$. Relative transcript levels were analyzed and normalized using $\mathrm{LjUbi-}$ quitin and LjATPase for L. japonicus genes and MtActin and MtRBP1 for Medicago truncatula genes. Three independent biological replicates for each sample were performed.

\section{ACKNOWLEDGMENTS}

We thank F. Xie (Shanghai Institute of Plant Physiology \& Ecology) for providing Rhizobium Sm2011 expressing LacZ and symbiosis defective mutant seeds (nfr5-3, ccamk-3, nsp2-2, nin-2), the Centre for Carbohydrate Recognition and Signaling (CARB) for providing LORE1 symrk-409 (30010361) mutant seeds, and the Samuel Roberts Noble Foundation for providing Tnt1 insertion mutant seeds.

\section{LITERATURE CITED}

Arrighi, J. F., Barre, A., Ben Amor, B., Bersoult, A., Soriano, L. C., Mirabella, R., de Carvalho-Niebel, F., Journet, E. P., Ghérardi, M., Huguet, T., Geurts, R., Dénarié, J., Rougé, P., and Gough, C. 2006. The Medicago truncatula lysin [corrected] motif-receptor-like kinase gene family includes NFP and new nodule-expressed genes. Plant Physiol. 142:265-279.

Bailly, A., Sovero, V., Vincenzetti, V., Santelia, D., Bartnik, D., Koenig, B. W., Mancuso, S., Martinoia, E., and Geisler, M. 2008. Modulation of P-glycoproteins by auxin transport inhibitors is mediated by interaction with immunophilins. J. Biol. Chem. 283:21817-21826.

Broghammer, A., Krusell, L., Blaise, M., Sauer, J., Sullivan, J. T., Maolanon, N., Vinther, M., Lorentzen, A., Madsen, E. B., Jensen, K. J., Roepstorff, P., Thirup, S., Ronson, C. W., Thygesen, M. B., and Stougaard, J. 2012. Legume receptors perceive the rhizobial lipochitin oligosaccharide signal molecules by direct binding. Proc. Natl. Acad. Sci. U.S.A. 109:13859-13864.

Cheng, X., Wang, M., Lee, H.-K., Tadege, M., Ratet, P., Udvardi, M. Mysore, K. S., and Wen, J. 2014. An efficient reverse genetics platform in the model legume Medicago truncatula. New Phytol. 201:1065-1076.

Cooper, J. E. 2007. Early interactions between legumes and rhizobia: Disclosing complexity in a molecular dialogue. J. Appl. Microbiol. 103: 1355-1365.

Di Pietro, A., Conseil, G., Pérez-Victoria, J. M., Dayan, G., BaubichonCortay, H., Trompier, D., Steinfels, E., Jault, J. M., de Wet, H., Maitrejean, M., Comte, G., Boumendjel, A., Mariotte, A. M., Dumontet, C., McIntosh, D. B., Goffeau, A., Castanys, S., Gamarro, F., and Barron, D. 2002. Modulation by flavonoids of cell multidrug resistance mediated by P-glycoprotein and related ABC transporters. Cell. Mol. Life Sci. 59: 307-322.

Falcone Ferreyra, M. L., Rius, S. P., and Casati, P. 2012. Flavonoids: Biosynthesis, biological functions, and biotechnological applications. Front. Plant Sci. 3:222.

Ferrer, J. L., Austin, M. B., Stewart, C., Jr., and Noel, J. P. 2008. Structure and function of enzymes involved in the biosynthesis of phenylpropanoids. Plant Physiol. Biochem. 46:356-370.

Geldner, N., Friml, J., Stierhof, Y.-D., Jürgens, G., and Palme, K. 2001. Auxin transport inhibitors block PIN1 cycling and vesicle trafficking. Nature 413:425-428.

Guo, L., and Paiva, N. L. 1995. Molecular cloning and expression of alfalfa (Medicago sativa L.) vestitone reductase, the penultimate enzyme in medicarpin biosynthesis. Arch. Biochem. Biophys. 320:353-360.
Ibáñez, F., Wall, L., and Fabra, A. 2017. Starting points in plant-bacteria nitrogen-fixing symbioses: Intercellular invasion of the roots. J. Exp. Bot. 68:1905-1918.

Kang, H., Xiao, A., Huang, X., Gao, X., Yu, H., He, X., Zhu, H., Hong, Z., and Zhang, Z. 2015. A Lotus japonicus cochaperone protein interacts with the ubiquitin-like domain protein CIP73 and plays a negative regulatory role in nodulation. Mol. Plant-Microbe Interact 28:534-545.

Kang, H., Zhu, H., Chu, X., Yang, Z., Yuan, S., Yu, D., Wang, C., Hong, Z. and Zhang, Z. 2011. A novel interaction between CCaMK and a protein containing the Scythe_N ubiquitin-like domain in Lotus japonicus. Plant Physiol. 155:1312-1324.

Kavanagh, K. L., Jörnvall, H., Persson, B., and Oppermann, U. 2008. Medium- and short-chain dehydrogenase/reductase gene and protein families: The SDR superfamily: Functional and structural diversity within a family of metabolic and regulatory enzymes. Cell. Mol. Life Sci. 65:3895-3906.

Ke, D., Fang, Q., Chen, C., Zhu, H., Chen, T., Chang, X., Yuan, S., Kang, H., Ma, L., Hong, Z., and Zhang, Z. 2012. The small GTPase ROP6 interacts with NFR5 and is involved in nodule formation in Lotus japonicus. Plant Physiol. 159:131-143.

Ke, D., Li, X., Han, Y., Cheng, L., Yuan, H., and Wang, L. 2016. ROP6 is involved in root hair deformation induced by Nod factors in Lotus japonicus. Plant Physiol. Biochem. 108:488-498.

Kelly, S., Sullivan, J. T., Kawaharada, Y., Radutoiu, S., Ronson, C. W., and Stougaard, J. 2018. Regulation of Nod factor biosynthesis by alternative NodD proteins at distinct stages of symbiosis provides additional compatibility scrutiny. Environ. Microbiol. 20:97-110.

Leong, S. A., Ditta, G. S., and Helinski, D. R. 1982. Heme biosynthesis in Rhizobium. Identification of a cloned gene coding for deltaaminolevulinic acid synthetase from Rhizobium meliloti. J. Biol. Chem. 257:8724-8730.

Li, H., Chen, M., Duan, L., Zhang, T., Cao, Y., and Zhang, Z. 2018. Domain swap approach reveals the critical roles of different domains of SYMRK in root nodule symbiosis in Lotus japonicus. Front. Plant Sci. 9:697.

Limpens, E., Franken, C., Smit, P., Willemse, J., Bisseling, T., and Geurts, R. 2003. LysM domain receptor kinases regulating rhizobial Nod factorinduced infection. Science 302:630-633.

Liu, C. W., and Murray, J. D. 2016. The role of flavonoids in nodulation host-range specificity: An update. Plants Basel 5:33.

López-Lara, I. M., van den Berg, J. D. J., Thomas-Oates, J. E., Glushka, J. Lugtenberg, B. J. J., and Spaink, H. P. 1995. Structural identification of the lipo-chitin oligosaccharide nodulation signals of Rhizobium loti. Mol. Microbiol. 15:627-638.

Madsen, E. B., Antolín-Llovera, M., Grossmann, C., Ye, J., Vieweg, S., Broghammer, A., Krusell, L., Radutoiu, S., Jensen, O. N., Stougaard, J., and Parniske, M. 2011. Autophosphorylation is essential for the in vivo function of the Lotus japonicus Nod factor receptor 1 and receptormediated signalling in cooperation with Nod factor receptor 5. Plant J. 65:404-417.

Madsen, E. B., Madsen, L. H., Radutoiu, S., Olbryt, M., Rakwalska, M. Szczyglowski, K., Sato, S., Kaneko, T., Tabata, S., Sandal, N., and Stougaard, J. 2003. A receptor kinase gene of the LysM type is involved in legume perception of rhizobial signals. Nature 425:637-640.

Madsen, L. H., Tirichine, L., Jurkiewicz, A., Sullivan, J. T., Heckmann, A. B., Bek, A. S., Ronson, C. W., James, E. K., and Stougaard, J. 2010. The molecular network governing nodule organogenesis and infection in the model legume Lotus japonicus. Nat. Commun. 1:10.

Maekawa, T., Kusakabe, M., Shimoda, Y., Sato, S., Tabata, S., Murooka, Y. and Hayashi, M. 2008. Polyubiquitin promoter-based binary vectors for overexpression and gene silencing in Lotus japonicus. Mol. PlantMicrobe Interact 21:375-382.

Martens, S., Preuss, A., and Matern, U. 2010. Multifunctional flavonoid dioxygenases: Flavonol and anthocyanin biosynthesis in Arabidopsis thaliana L. Phytochemistry 71:1040-1049.

Mulligan, J. T., and Long, S. R. 1985. Induction of Rhizobium meliloti nodC expression by plant exudate requires nodD. Proc. Natl. Acad. Sci. U.S.A. 82:6609-6613.

Ng, J. L., Hassan, S., Truong, T. T., Hocart, C. H., Laffont, C., Frugier, F., and Mathesius, U. 2015. Flavonoids and auxin transport inhibitors rescue symbioticnodulation in the Medicago truncatula cytokinin perception mutant cre1. Plant Cell 27:2210-2226.

Oldroyd, G. E., Murray, J. D., Poole, P. S., and Downie, J. A. 2011. The rules of engagement in the legume-rhizobial symbiosis. Annu. Rev. Genet. 45:119-144.

Peer, W. A., Bandyopadhyay, A., Blakeslee, J. J., Makam, S. N., Chen, R. J., Masson, P. H., and Murphy, A. S. 2004. Variation in expression and protein localization of the PIN family of auxin efflux facilitator proteins 
in flavonoid mutants with altered auxin transport in Arabidopsis thaliana. Plant Cell 16:1898-1911.

Radutoiu, S., Madsen, L. H., Madsen, E. B., Felle, H. H., Umehara, Y., Grønlund, M., Sato, S., Nakamura, Y., Tabata, S., Sandal, N., and Stougaard, J. 2003. Plant recognition of symbiotic bacteria requires two LysM receptor-like kinases. Nature 425:585-592.

Radutoiu, S., Madsen, L. H., Madsen, E. B., Jurkiewicz, A., Fukai, E., Quistgaard, E. M. H., Albrektsen, A. S., James, E. K., Thirup, S., and Stougaard, J. 2007. LysM domains mediate lipochitin-oligosaccharide recognition and $N f r$ genes extend the symbiotic host range. EMBO J. 26: 3923-3935.

Santelia, D., Henrichs, S., Vincenzetti, V., Sauer, M., Bigler, L., Klein, M., Bailly, A., Lee, Y., Friml, J., Geisler, M., and Martinoia, E. 2008. Flavonoids redirect PIN-mediated polar auxin fluxes during root gravitropic responses. J. Biol. Chem. 283:31218-31226.

Schäfer, A., Tauch, A., Jäger, W., Kalinowski, J., Thierbach, G., and Pühler, A. 1994. Small mobilizable multi-purpose cloning vectors derived from the Escherichia coli plasmids pK18 and pK19: Selection of defined deletions in the chromosome of Corynebacterium glutamicum. Gene 145:69-73.

Shimoda, Y., Mitsui, H., Kamimatsuse, H., Minamisawa, K., Nishiyama, E., Ohtsubo, Y., Nagata, Y., Tsuda, M., Shinpo, S., Watanabe, A., Kohara, M., Yamada, M., Nakamura, Y., Tabata, S., and Sato, S. 2008. Construction of signature-tagged mutant library in Mesorhizobium loti as a powerful tool for functional genomics. DNA Res. 15:297-308.

Simon, R., Priefer, U., and Pühler, A. 1983. A broad host range mobilization system for in vivo genetic engineering: Transposon mutagenesis in gram negative bacteria. Bio/Technology 1:784-791.

Smit, P., Limpens, E., Geurts, R., Fedorova, E., Dolgikh, E., Gough, C., and Bisseling, T. 2007. Medicago LYK3, an entry receptor in rhizobial nodulation factor signaling. Plant Physiol. 145:183-191.

Sørensen, K. K., Simonsen, J. B., Maolanon, N. N., Stougaard, J., and Jensen, K. J. 2014. Chemically synthesized 58-mer LysM domain binds lipochitin oligosaccharide. ChemBioChem 15:2097-2105.
Tadege, M., Wen, J., He, J., Tu, H., Kwak, Y., Eschstruth, A., Cayrel, A., Endre, G., Zhao, P. X., Chabaud, M., Ratet, P., and Mysore, K. S. 2008. Large-scale insertional mutagenesis using the Tnt1 retrotransposon in the model legume Medicago truncatula. Plant J. 54:335-347.

Waadt, R., Schmidt, L. K., Lohse, M., Hashimoto, K., Bock, R., and Kudla, J. 2008. Multicolor bimolecular fluorescence complementation reveals simultaneous formation of alternative CBL/CIPK complexes in planta. Plant J. 56:505-516.

Wang, C., Zhu, M., Duan, L., Yu, H., Chang, X., Li, L., Kang, H., Feng, Y., Zhu, H., Hong, Z., and Zhang, Z. 2015. Lotus japonicus clathrin heavy Chain1 is associated with Rho-Like GTPase ROP6 and involved in nodule formation. Plant Physiol. 167:1497-1510.

Wang, L., Wang, L., Tan, Q., Fan, Q., Zhu, H., Hong, Z., Zhang, Z., and Duanmu, D. 2016. Efficient inactivation of symbiotic nitrogen fixation related genes in Lotus japonicus using CRISPR-Cas9. Front. Plant Sci. 7:1333.

Wang, X. 2011. Structure, function, and engineering of enzymes in isoflavonoid biosynthesis. Funct. Integr. Genomics 11:13-22.

Wasson, A. P., Pellerone, F. I., and Mathesius, U. 2006. Silencing the flavonoid pathway in Medicago truncatula inhibits root nodule formation and prevents auxin transport regulation by rhizobia. Plant Cell 18:1617-1629.

Weston, L. A., and Mathesius, U. 2013. Flavonoids: Their structure, biosynthesis and role in the rhizosphere, including allelopathy. J. Chem. Ecol. 39:283-297.

Xie, F., Murray, J. D., Kim, J., Heckmann, A. B., Edwards, A., Oldroyd, G. E., and Downie, J. A. 2012. Legume pectate lyase required for roo infection by rhizobia. Proc. Natl. Acad. Sci. U.S.A. 109:633-638.

Zhang, J., Subramanian, S., Stacey, G., and Yu, O. 2009. Flavones and flavonols play distinct critical roles during nodulation of Medicago truncatula by Sinorhizobium meliloti. Plant J. 57:171-183.

Zhu, H., Chen, T., Zhu, M., Fang, Q., Kang, H., Hong, Z., and Zhang, Z. 2008. A novel ARID DNA-binding protein interacts with SymRK and is expressed during early nodule development in Lotus japonicus. Plant Physiol. 148:337-347. 